\title{
Associative priming in perceptual identification: Effects of prime-processing requirements
}

\author{
JENNIFER S. BURT \\ University of Queensland, Brisbane, Queensland, Australia \\ MICHAEL B. WALKER \\ Griffith University, Brisbane, Queensland, Australia \\ MICHAEL S. HUMPHREYS \\ University of Queensland, Brisbane, Queensland, Australia \\ and \\ GERALD TEHAN \\ University of Southern Queensland, Toowoomba, Queensland, Australia
}

\begin{abstract}
Three experiments assessed the effects of prime-processing instructions on associative priming in word identification and episodic memory for primes. In Experiment 1, groups instructed to read the prime silently or generate silently an associate of the prime showed a larger accuracy benefit for related over unrelated targets than did a group that decided whether an asterisk was to the right or left of the prime. The asterisk-search group showed a weaker repetition effect on a subsequent identification test of primes, indicating that the weaker priming in this group was a result of poorer perceptual processing. On a cued-recall test for primes, the generate group was superior to the other groups. In Experiment 2, we found that with weak prime-target associations, priming was comparable for read and generate groups and stronger than estimated for a guessing strategy, on the basis of single predictions made from each prime by an additional group. In Experiment 3, we demonstrated that the read and generate instructions produced similar mispriming and inhibitory effects. The results suggest that the depths of prime-processing manipulations do not have parallel effects on priming and episodic memory, and that associative priming in word identification, as in other tasks, may involve an expectancy process.
\end{abstract}

The seminal work on levels of processing (Craik \& Lockhart, 1972; Craik \& Tulving, 1975) led to the conclusion that more semantic processing of to-beremembered material produces better recall or episodic recognition than does superficial processing. Recently, this generalization has been interpreted within more general frameworks that relate the nature of processing occurring at study and at test. For example, Roediger, Weldon, and Challis (1989) have accumulated evidence in favor of a distinction between conceptual and data-driven (perceptual) processing, with transfer between tasks to be expected to the extent that the processing operations at test recapitulate those involved in the study phase (cf. transfer-appropriate processing, Morris, Bransford, \& Franks, 1977).

The present research focuses on the semantic priming phenomenon in word recognition. A parallel in the lexical memory domain to the levels-of-processing effect has been obtained in demonstrations that deeper processing

The research reported here was supported by Grant 634 from the Australian Research Grants Scheme. Please address correspondence to J. S. Bur, Department of Psychology, University of Queensland, Qld, 4072, Australia. of a prime word increases the magnitude of the semantic priming effect (e.g., Becker, 1979; Fischler \& Goodman, 1978; Meyer \& Schvaneveldt, 1971). For example, Smith, Theodor, and Franklin (1983) showed in a lexical decision task that the reduction in correct response latency to a target word (e.g., QUEEN) produced by the prior presentation of a related prime (KING) was greater when the prime was processed semantically (e.g., whether the prime denoted a living thing) than when more superficial processing (e.g., silent reading, letter search) was done on the prime. These results have been replicated in the lexical decision task (Henik, Friedrich, \& Kellogg, 1983) as well as in color naming (Henik et al., 1983; Parkin, 1979) and pronunciation (Parkin, 1984).

However, the utility of levels of processing as a theoretical framework has been limited by the difficulty in providing assessments of processing depth that are independent of the memory test (cf. Baddeley, 1990). Consequently, it is not clear whether depth-of-processing effects reflect variations along a psychologically significant "depth" dimension, or whether different kinds of processing show a different pattern of graded effects across memory paradigms. In some studies of priming, this issue has been addressed via the inclusion of a postpriming memory test on primes. 
Consistent with a common mechanism for depth-ofprocessing effects, Smith et al. (1983) found that groups given an unexpected episodic recognition test showed graded memory for primes (but not targets) that paralleled priming effects over levels-of-prime-processing conditions. Also, Parkin $(1979,1984)$ found superior free recall of primes in a semantic prime-processing group over a nonsemantic processing group. The present experiments extend the investigation of depth of processing in associative priming to the perceptual identification task, which has been used infrequently to assess semantic priming. The primary aim was to test the generality of the above evidence for parallel depth-of-processing effects in semantic priming and conceptually driven tests used in episodic memory research.

Semantic priming effects were initially interpreted in terms of an automatic spread of excitation from the prime's node in a semantic network to nearby nodes for related words (cf. Collins \& Loftus, 1975). Subsequently, priming research has provided evidence for the involvement of attentional or strategic factors. For example, at long prime-target stimulus onset asynchronies (SOAs), Neely (1977) produced an attentional priming effect with previously unrelated primes and targets that the subjects expected to co-occur during the experimental session. The priming effect consisted of facilitation for related targets and inhibition for unrelated targets relative to a neutral prime condition, and was interpreted in terms of expectancies about the target that were generated during prime processing (see also Becker, 1980; Burke, White, \& Diaz, 1987; den Heyer, Briand, \& Smith, 1985; Tweedy \& Lapinski, 1981; Tweedy, Lapinski, \& Schvaneveldt, 1977).

In the lexical decision task, there is evidence for the operation of an additional, retrospective strategy in which subjects check the prime and target for relatedness, with a consequent bias toward a "yes" decision for related pairs and a "no" decision for unrelated pairs (de Groot, 1984, 1985; den Heyer, 1985; Lorch, Balota, \& Stamm, 1986; Seidenberg, Waters, Sanders, \& Langer, 1984; Stanovich \& West, 1983). This strategy is not supported in tasks requiring an identification of a target, such as the perceptual identification task used in the present experiments.

Depth-of-prime-processing effects have been interpreted both in terms of spread of activation (e.g., Smith et al., 1983) and attentional mechanisms (e.g., Henik et al., 1983). Bentin and Katz (1984) argued, consistent with an automatic spread-of-activation account, that priming occurs whenever subjects have recognized a related prime. The smaller priming effect that has been observed under superficial prime-processing conditions was thought by Bentin and Katz to reflect a failure to read the prime "as a whole word" on some or all trials. Contrary to Bentin and Katz, Smith et al. (1983) showed that animateinanimate judgments about the prime produced greater priming than did silent reading of the prime. However, this depth-of-prime-processing effect with recognized primes would be more convincing if the authors had veri- fied that subjects in the silent reading condition were reliably reading primes. In the present experiments, perceptual processing of primes was assessed after priming trials.

Our predictions for the present experiment were guided by the sequential intersection model of Humphreys, Wiles, and Bain (1992), which provides an expectancy-based account of priming. As an account of semantic priming, the model shares essential features with the expectancy theories of Becker $(1980,1985)$. Expectancies are generated when subjects process a word associatively, with implicit, parallel activation of associates of the word. The typical "semantic" priming effect is assumed to depend upon associative processing of the prime with nonassociative processing of the target. Thus, the prime CAT produces DOG, FUR, LICK, and so on, and the target DOG produces itself and perhaps perceptual relatives. The information activated by the prime and the target is combined in an intersection process, from which the target DOG emerges. When the prime is unrelated to the target, the intersection of word sets activated by prime and target is empty, and if the subject is relying on information provided by the prime, inhibition is observed. The extent to which subjects process primes associatively and rely on the information yielded by the intersection process is influenced by the salience of the prime-target relationship and the ambiguity of perceptual information about the target.

In the perceptual identification task, subjects are likely to rely on information provided by the prime. Therefore, it was of interest whether the depth-of-prime-processing effects observed in lexical decision and word- and colornaming tasks would be observed. In the first of the present studies, three prime-processing conditions were used to cover a range of points on a semantic processing dimension. In a superficial processing condition, subjects decided (covertly) whether an asterisk was located to the right or left of the prime. An intermediate semantic processing condition required the subjects to read the prime silently. In a high-semantic condition, they covertly generated an associate of the prime word. No overt response to the prime was required, because we were concerned that an overt response might interfere with subsequent target processing. Although this is of more concern with latency measures than accuracy measures, we hoped to extend our procedures to lexical decision and naming tasks.

Two tests of prime processing, administered after priming, were designed to provide a set of "marker" variables that could be used to assess correspondences between the magnitude of priming and perceptual and conceptual processing of the prime. The first test, cued recall, assessed episodic memory for primes. The cue was an associate of the prime that had not been presented during the priming session (cf. Humphreys, Bain, \& Pike, 1989; Nelson \& McEvoy, 1979). This task, which fits the criteria of a conceptually driven test (Roediger et al., 1989), has been found to be sensitive to variations in depth of processing, as demonstrated by Humphreys, Bain, Sweeton, and Pacey (1991). We expected cued recall to vary directly with our three prime-processing conditions, with 
the generate group showing the highest and the asterisksearch group the lowest performance. The second test assessed the repetition benefit in perceptual identification for primes. The increase in accuracy or speed of recognition of a repeated word is a well-established phenomenon (e.g., Evett \& Humphreys, 1981; Forster \& Davis, 1984; Jacoby \& Dallas, 1981; Jacoby \& Witherspoon, 1982; Kirsner, Milech, \& Standen, 1983; Scarborough, Cortese, \& Scarborough, 1977). The repetition effect in lexical decision, naming, and identification tasks depends upon recognition of the word on its first presentation, but unlike cued recall, is relatively insensitive to semantic processing (Jacoby, 1983; Jacoby \& Dallas, 1981). Instead, it appears to depend upon the subject's involvement with the physical features of the word (Jacoby, 1983), and fits the criteria specified by Roediger et al. (1989) for a datadriven test. The inclusion of a repetition test on primes provides a measure that should be related to the adequacy of perceptual processing of the prime (cf. Hayman \& Jacoby, 1989). Thus, it enables an objective specification of Bentin \& Katz's (1984) suggestion that subjects read the prime "as a whole word."

If the parallelism observed by Smith et al. (1983) and Parkin (1979, 1984) across semantic priming and conceptual tests of episodic memory for primes extends to perceptual identification, priming effects will covary with cued-recall performance. This prediction would also be made if the generation condition induces the subjects to use a prediction strategy. However, variations in priming in perceptual identification (unlike lexical decision; cf. Smith et al., 1983) may more closely correspond to the pattern of repetition effects, on the grounds that in conditions fostering recognition of the prime, the subjects will notice the relevance of the prime and will rely on the prime in identifying the target.

\section{EXPERIMENT 1}

\footnotetext{
Method

Subjects. Seventy-two first-year psychology undergraduates of both sexes served as subjects for course credit. They were randomly allocated in equal numbers to three groups, generate, read, and asterisk search, formed on the basis of prime-processing instructions.

Materials and Design. The experiment was conducted in four phases. In the first, involving perceptual identification of single words, a stimulus duration supporting $50 \%$ accuracy was determined for each subject for targets in the priming phase. Priming was assessed in the second phase. The subjects were presented with $\mathbf{2 0}$ related and $\mathbf{4 0}$ unrelated trials. For half of the unrelated trials, the primes were designated to-be-retrieved words for the cued-recall test. The third phase was the repetition test of primes from related and unrelated pairs used to assess priming in Phase 2. The fourth phase was the cued-recall test for the 20 primes from Phase 2, with cues for the primes provided by their strong associate primes (not presented in the priming session).

Stimulus words, four to seven letters long, were chosen on the basis of pilot work by the second author. They included 100 words for duration determination and 24 randomly paired words for practice before priming trials. Sixty associate pairs from the Thomson, Meredith, and Browning Australian word association norms (1976) served as priming items. Target mean frequency was 100 in the
}

Kucera and Francis corpus (1967). The mean association strength for the pairs (proportion of the norm sample giving the target as the first associate of the prime) was $28 \%$.

The priming session consisted of 20 related trials, 20 unrelated trials and, for the purposes of the cued-recall test, 20 "new-target" unrelated trials. For each subject, the set of 60 associate pairs was randomly divided into three equal subsets to provide 20 pairs for each of the three trial types. For related and unrelated trials, the primes and target pairs were intact and randomly re-paired, respectively. For new-target trials, the primes were randomly paired with 20 targets taken at random for each subject from an additional set of 40 words (mean frequency 91 , Kucera \& Francis, 1967) that were taken from the Thomson et al. (1976) norms. The trials were presented in a random sequence with the constraint that no more than 3 related trials occurred in succession and no more than 6 consecutive trials occurred without a related trial.

For the 60 prime repetition trials, there were 40 old primes, 20 from each of the related and unrelated prime conditions, and 20 new words. The latter were the words from the additional 40-word set that had not been used for new-target priming trials. For cued recall, the subjects were required to recall the primes shown in newtarget pairs in the priming phase. The cues provided were the 20 related targets that had been replaced with unrelated words. Thus, for the new-target pair TIME-CRISIs, a subject was given CLOCK as a cue to recall TIME.

Procedure. The subjects sat in front of an Amiga 1000 microcomputer with an Amiga NSTC monitor. They were informed that there were several parts to the experiment. They read an instruction sheet prior to each phase of the experiment, supplemented by explanation where necessary. The words were presented on a blue background in uppercase white letters, subtending a visual angle of approximately $.50^{\circ}$. Prime words for the asterisk-search group had an asterisk immediately to the left or right of the word. It is unlikely that the absence of the asterisk for the other conditions contributed to the priming results, because Smith (1979) found that the presence above the prime of the target letter for a letter search had no effect on subjects who were required to read the prime. Each word to be identified by the subject was followed by a mask, composed of eight randomly selected uppercase consonants.

In the preliminary duration determination phase, the subjects saw words in blocks of 10 , with an interval of 4 sec between trials. The mask was presented for $100 \mathrm{msec}$, timed from word offset. The subject was informed about the random-letter mask and was asked to name the briefly presented word or respond "no" if unable to do so. For the first block of 10 trials, the word exposure duration was set at $100 \mathrm{msec}$. In subsequent blocks, the duration was rectuced by $20 \mathrm{msec}$ (to a limit of $60 \mathrm{msec}$ ) after each block when the subject scored $70 \%$ correct or better, was increased by $10 \mathrm{msec}$ when performance was at or below $30 \%$ correct for two consecutive blocks, or otherwise remained unaltered. When the stimulus duration reached $60 \mathrm{msec}$, decreases in duration were made in steps of $10 \mathrm{msec}$. The duration set for Block 11 was used for the words to be identified in the priming and repetition phases.

In the priming phase, which followed immediately after the duration determination phase, the subjects identified target words and processed the prime words according to condition. Generate subjects were to think silently of a word closely associated with the prime. Read subjects were to read the prime silently, and asterisksearch subjects were to decide whether an asterisk presented adjacent to the prime was presented to the left or the right of the word. No overt response was required

The primes were presented for $1 \mathrm{sec}$, with a 1-sec pause following. The target was presented at the duration previously determined for the subject, and a mask was presented for $100 \mathrm{msec}$ after the target, as described previously. There was a pause of 4 sec between mask offset and the next trial. Errors in target identification were manually recorded by the experimenter. 
The repetition phase followed. The subjects were instructed that the task was identical to the first task (duration determination). There were 60 word identification trials at the temporal parameters set at the end of Phase 1. Errors were manually recorded. The cuedrecall test was administered last. The subjects were given $5 \mathrm{~min}$ to complete the test, which required them to write next to each of 20 cue words a word that was presented for $1 \mathrm{sec}$ in the second part of the experiment. They were informed that the cue words might "remind" them of the to-be-remembered words.

\section{Results}

The mean $50 \%$ identification exposure durations for the generate, read, and asterisk-search groups were in the vicinity of $40 \mathrm{msec}^{1}{ }^{1}$ The mean proportion of correct target identifications by group and condition for the priming phase are shown in Table 1. Analyses over subjects were performed to test differences in priming, repetition, and cued recall for the three groups. Item analyses were not performed, because each subject had a different sample of the 60 paired associates for each of the trial types and, therefore, item differences were confounded with subject differences.

For the priming phase, the data for the new-target condition were excluded from analysis because the target items for these trials came from an item pool prepared separately from the set used for related and unrelated trials. Table 1 shows that the results for new-target trials are similar to those for unrelated trials. A trial type $x$ group analysis of variance (ANOVA) on accuracy scores for related and unrelated trials revealed no main effect for group $(F<1)$, a significant priming facilitation for targets following related versus unrelated primes $[F(2,69)=215, p<.001]$, and a significant group $\times$ trial type interaction $[F(2,69)=6.3, p<.005]$. A Tukey's honestly significant difference $(H S D)$ test of priming effects showed that, although all priming effects were significant, the read and generate groups had greater priming effects than the asterisk-search group $(p<.05)$, and the generate and read groups did not differ on the magnitude of priming. On related trials, both the generate and read groups were more accurate than the asterisk-search group (Tukey's $H S D, p<.05$ ), whereas for unrelated trials the only significant difference was the inferior performance of the generate compared with the asterisksearch group (Tukey's $H S D, p<.05$ ).

The accuracy data for the prime repetition test are shown in Table 2. Because there was no difference in accuracy between primes seen in related versus unrelated

Table 1

Mean Proportions of Correct Target Identifications During the Priming Phase for the Related and Two Unrelated Conditions in Experiment 1

Prime-Processing Group

\begin{tabular}{|c|c|c|c|c|c|c|}
\hline \multirow{3}{*}{$\begin{array}{c}\text { Prime } \\
\text { Condition }\end{array}$} & \\
\hline & \multicolumn{2}{|c|}{ Generate } & \multicolumn{2}{|c|}{ Read } & \multicolumn{2}{|c|}{ Asterisk-Search } \\
\hline & $M$ & $S D$ & $M$ & $S D$ & $M$ & $S D$ \\
\hline Related & .79 & .13 & .78 & .12 & .70 & .14 \\
\hline Unrelated & .31 & .15 & .39 & .20 & .44 & .20 \\
\hline New Target & .33 & .16 & .37 & .21 & .43 & .21 \\
\hline
\end{tabular}

Table 2

Mean Proportions of Correct Identifications in the Repetition Test for Old Primes (Pooled Over Related and Unrelated) and New Words in Experiment 1

\begin{tabular}{|c|c|c|c|c|c|c|}
\hline \multirow{3}{*}{$\begin{array}{l}\text { Word } \\
\text { Type }\end{array}$} & \multicolumn{6}{|c|}{ Prime-Processing Group } \\
\hline & \multicolumn{2}{|c|}{ Generate } & \multicolumn{2}{|c|}{ Read } & \multicolumn{2}{|c|}{ Asterisk-Search } \\
\hline & $M$ & $S D$ & $M$ & $S D$ & $M$ & $S D$ \\
\hline Old & .60 & .13 & .66 & .14 & .57 & .15 \\
\hline New & .34 & .14 & .43 & .18 & .42 & .17 \\
\hline
\end{tabular}

pairs during priming $(F<1)$, these items were pooled to form a set of old primes. An ANOVA for group $x$ word type (old primes vs. new words) revealed no main effect for group $[F(2,69)=1.89, p>.05]$, a significant difference between old and new words, and a significant group $\times$ word type interaction $[F(1,69)=164.4, p<$ .001 and $F(2,69)=4.63, p<.05$, respectively]. Tukey's $H S D$ indicates that both the generate and read groups showed greater old-new differences than did the asterisk-search group $(p<.05)$, with the generate and read groups not differing. However, the old-new difference was reliable for the asterisk-search group (Tukey's $H S D, p<.01)$.

The percentages of primes produced in the cued-recall phase for the generate, read, and asterisk-search groups were $28.3 \%, 19.8 \%$, and $17.9 \%$, respectively. Planned comparisons revealed reliable differences between the generate and read groups $[F(1,69)=4.7, p<.05]$ and between the generate and asterisk-search groups $[F(1,69)=6.9, p<.05]$. The read and asterisk-search groups did not differ $(F<1)$.

\section{Discussion}

The priming effects on identification were evident for all prime-task groups, with the generate and read groups showing stronger priming than the asterisk-search group. In addition, all the groups showed a repetition benefit in the Phase 2 word identification test of old primes and new words, with the generate and read groups again showing a greater effect than the asterisk-search group. On the final cued-recall test for primes, the generate subjects were superior to both the read and asterisk-search subjects. The discrepancy between the cued-recall and repetition results is consistent with the findings of Jacoby (1983) that repetition in perceptual identification is not sensitive to semantic or elaborative processing on the first presentation. In addition, it exemplifies the dissociations that have been observed between conceptual and data-driven memory tests (cf. Roediger et al., 1989).

The cued-recall results indicate that, at least on some trials, the generate subjects were performing the associategeneration task as instructed, and that there was a difference in the depth of semantic processing between the generate and read groups. This difference in semantic processing, however, did not produce a difference in priming between these groups. In correspondence with their priming results, the generate and read groups showed 
comparable prime repetition effects, suggesting that they engaged in similar amounts of perceptual processing of the primes. The poorer repetition benefit for the asterisksearch group relative to the other groups suggests less perceptual processing of primes in these subjects. (Because the new items in the repetition phase were not taken from the prime set, the precise magnitude of the repetition effect for each group is not known.) Consequently, the present results provide some support for Bentin and Katz's (1984) argument that smaller priming effects with superficial prime-processing tasks are attributable to inadequate perceptual processing of primes (failure to process primes as "whole words"), rather than a lack of semantic processing.

The demonstration of a repetition effect for primes in the asterisk-search condition indicates some whole-word processing of primes, but we cannot rule out the possibility that some primes received full processing and others received attenuated processing. Presumably, the priming that was observed in this group is an indication either of the extent to which the asterisk-search task is compatible with reading the prime, or the extent to which the subjects were disobeying instructions and reading the prime rather than making an asterisk search. Perhaps a number of factors, including SOA, target task, and for asterisk searches, distance of the asterisk from the prime, may affect the likelihood that primes are read. Interestingly, Besner, Smith, and MacLeod (1990) recently showed that when their previously used letter-search task was modified to require subjects to judge whether two words shared a common letter, evidence of lexical and semantic activation was obtained. They suggested that the task used in the earlier Smith et al. (1983) study encouraged subjects to make a visual match at a prelexical level. Conversely, Bentin and Katz (1984) observed comparable priming in groups making a case judgment and a lexical decision about the prime, but this effect was not replicated by Kaye and Brown (1985), who observed priming for lexical decisions but not case judgments. The long prime-target SOAs used by Bentin and Katz (more than $2,300 \mathrm{msec}$ ) may have encouraged attention to the prime. Although we do not know when the asterisk-search subjects were reading the prime, their results are clear-cut in that weaker priming was accompanied by evidence that the subjects were not consistently reading the primes. Consequently, no further investigation of this condition was undertaken.

It is more interesting that the generation of an associate to the prime by the generate subjects did not produce more priming than that observed in the subjects merely reading the prime. This result is inconsistent with previous lexical decision research showing greater priming with semantic prime-processing instructions than with silent reading of the prime (e.g., Smith et al., 1983). It is likely that, as suggested earlier, the subjects relied heavily on the prime in both the read and generate conditions. One implication of the similarity of the priming results for these two groups is that the associate-generation task does not produce activity that contributes to priming. In other words, making a single explicit prediction that can be matched with the target stimulus information does not assist in target identification.

Alternatively, however, it is possible that there was a difference between the two groups in the magnitude of priming that our experiment failed to detect. For example, because the two groups displayed high accuracy on related trials, any difference may be masked by a nearceiling improvement in identification. The trend toward inferior performance on unrelated trials by the generate relative to the read subjects is indicative of a possible differential inhibitory effect, and hence supports this possibility. Unfortunately, it is also arguable that the unrelated prime results for the generate group arise not from an inhibitory process but from an overall inferiority at the perceptual identification task in this group of subjects. It is noteworthy that during the repetition test, the generate group performed more poorly on new words than the remaining two groups. This result may reflect the differential use of retrieval or other strategies in generate subjects; for example, a heavier reliance on "conceptual" than "data-driven" processing that has transferred from the priming session. However, the overall pattern of results plausibly suggests a lower ability in word identification in this group. Experiment 2 was designed to investigate further the effect of generating an associate to the prime on the magnitude of priming.

\section{EXPERIMENT 2}

The associative strength of prime-target pairs, and therefore target predictability, was varied over two levels (high and low) within subjects. Three between-subjects conditions were employed. The first two groups corresponded to the read and generate groups of Experiment 1 . A third, "guessing"' group was introduced to view the priming stimuli at a longer prime-target interval and predict, in writing, the target from the prime. The responses provided by the guessing subjects were used to estimate the probability of predicting targets from primes in the weak and strong associate conditions. Thus, by contrasting two levels of association and providing an independent estimate of the success of target prediction from primes, the design allows a more direct assessment of the extent to which priming effects in read and generate prime tasks are attributable to the use of a simple prediction strategy.

The read and generate groups were subsequently tested on cued recall for primes, as in Experiment 1. The prime repetition test, which did not distinguish the read and generate groups in Experiment 1, was omitted.

\section{Method}

Subjects. Seventy-two male and female first-year psychology students participated for course credit. They were randomly divided into three groups: generate, read, and guess $(n=24$ each).

Materials and Design. The read and generate groups had duration determination, priming, and cued-recall tasks. The guessing 
group participated only during the priming task. In the priming session, the subjects had equal proportions of weakly related, strongly related, and unrelated trials. The unrelated trials had strong primes randomly paired with targets that were matched on frequency and length distribution to related targets. Although the unrelated condition is an inappropriate baseline for comparison with weakly related pairs, in that the weak primes may differ from primes that occur in strong associate pairs, it was adequate to test for a difference in priming effects between the two groups. The unrelated-trial primes were tested in the cued-recall test, as described previously.

All words were four to six letters long. Sixty target words (mean frequency 100 , Kucera \& Francis, 1967) were selected with a weak and strong associative stimulus as a prime for each (mean frequency: 83 and 68 , respectively), with primes and targets approximately matched on length. The strong associative primes were taken from the Thomson et al. (1976) norms and the prime-target pairs had a mean associative strength of $28 \%$. The weak primes were taken from Thomson et al. (1976), Miller (1970), Palermo and Jenkins (1964), or were made up. The associative strengths for weak pairs taken from norms ranged from $1 \%$ to $8 \%, 5 \%$ to $10 \%$, and $2 \%$ to $17 \%$, respectively.

An additional set of 40 words, matched to the targets on mean length and frequency, provided targets for a new-target condition that served as an unrelated prime control. A further set of 48 unrelated words was divided into 24 primes and 24 targets that were randomly paired for each subject to make up an initial block of practice trials. Finally, 100 single words were used for stimulus duration determination, as described in Experiment 1.

Each subject in each of the three groups saw a randomly selected set of 20 targets with weak primes and a set of 20 different targets with strong primes. The strong primes for the remaining 20 targets were paired with 20 randomly selected unrelated targets from the additional pool of 40 words. The trials were presented in a random sequence with the constraint that no more than three "strong" trials occurred in succession and no more than six consecutive trials occurred without a strong trial.

For the cued-recall test, the normatively associated unpresented targets for the new-target primes were used as cues for the recall of the primes.

Procedure. For the read and generate conditions, the duration determination, priming, and cued-recall phases were conducted as in Experiment 1. The guessing group saw each prime for $1 \mathrm{sec}$ and its target, unmasked, for $.5 \mathrm{sec}$ on an Amiga display similar to that used for the read and generate groups. A 5-sec delay was interposed between prime offset and target onset. The subjects were in structed to "try to guess the next word" occurring after the prime, and to write their guess for each word on an answer sheet with numbered trials. They were informed that the words were sometimes related and sometimes unrelated. At the end of each trial, the trial number was shown on the screen. The intertrial interval was $4 \mathrm{sec}$.

\section{Results}

There was no difference between groups in the target durations used for the identification task. Priming phase data for the read and generate groups are shown in $\mathrm{Ta}$ ble 3. Because the new-target and unrelated prime conditions produced comparable performance in Experiment 1 , the new-target means were used to provide the unrelated condition scores in the statistical analyses. The new-target versus related prime trial comparison is confounded by item differences but allows unambiguous estimates of relative priming effects for the groups. A groups $x$ trial type ANOVA shows a significant difference for the three trial types $[F(2,92)=117.3, p<.001]$, no reliable group effect $(F<1)$, and a nonsignificant group $\times$
Table 3

Mean Proportions of Correct Identifications in the Priming

Phase for Each of the Three Trial Types in Experiment 2

\begin{tabular}{|c|c|c|c|c|}
\hline \multirow{3}{*}{$\begin{array}{c}\text { Prime } \\
\text { Condition }\end{array}$} & \multicolumn{4}{|c|}{ Prime-Processing Group } \\
\hline & \multicolumn{2}{|c|}{ Generate } & \multicolumn{2}{|c|}{ Read } \\
\hline & $M$ & $S D$ & $M$ & $S D$ \\
\hline Strong Association & .81 & .16 & .79 & .15 \\
\hline Weak Association & .69 & .14 & .67 & .20 \\
\hline Unrelated & .36 & .22 & .45 & .21 \\
\hline
\end{tabular}

trial interaction $[F(2,92)=2.6, p=.08]$. Tukey's $H S D$ test shows that for each group separately, all pairwise comparisons between the three trial types are significant $(p<.05)$. Table 3 shows that the read and generate groups performed similarly on related trials but that the generate subjects tended to be less accurate than the read subjects on unrelated trials. Some evidence for an inhibitory effect on new-target trials in the generate group relative to the read group was provided by a significant group $x$ trial type interaction when responses for weak and strong related trials were pooled $[F(1,92)=4.1, p<.05]$.

On cued recall for new-target primes, the generate subjects performed better than the read subjects [a mean proportion of .40 vs. .30 primes recalled; $F(1,46)=4.5$, $p<.05]$.

The responses for the guess subjects were scored according to the prime-target relation, with the mean proportion correct for strong associates, weak associates, and new-target pairs being $.35, .03$, and 0 , respectively $[F(2,46)=118.6, p<.001]$. The results for strong associates correspond quite closely with the mean associative strength of the pairs reported in the word association norms from which the words were selected $(.28)$, whereas the results for weak associates confirm that the associative strength for these pairs is low.

\section{Discussion}

The subjects who read the prime silently showed associative priming that was as strong as that shown by the subjects who silently generated an associate of the prime. In addition, both groups showed stronger facilitation of target recognition when the prime was a strong associate than when it was a weak associate. This result demonstrates a strength-of-association effect in priming that has been observed in pronunciation (see Neely, 1991) and color naming (Warren, 1974) but has been elusive in lexical decision experiments (see de Groot, Thomassen, \& Hudson, 1982; Koriat, 1981). Consistent with Experiment 1 , the two groups differed in cued recall, indicating that the generate group engaged in more elaborative or semantic processing of the prime than did the read group.

The weaker performance in the new-target condition on unrelated trials for the generate relative to the read group points to a possible inhibitory effect that was weaker or absent in the read group. Some evidence supports this conclusion. First, a similar trend was observed for this 
group in Experiment 1. Second, the proportion correct for new-target trials in these subjects (.36) clearly falls short of the .50 accuracy achieved during Phase 1 [duration determination, $t(1,23)=3.1, p<.01]$. However, the overall poor performance of the generate subjects in Experiment 1, and their unpredicted decline in that experiment from .50 in Phase 1 to .34 for new words in the repetition phase, throws doubt on this evidence. Consequently, no firm conclusion can be drawn about differential inhibitory effects for the generate and read groups. This issue is pursued in Experiment 3.

Although we cannot confidently draw inferences about inhibition on unrelated trials, the data provided by the guess group give a clear indication of the extent to which the read and generate subjects are using a simple predictand-match strategy for target identification on priming trials. Those data indicate that although the subjects were moderately successful at guessing the strong associate of a prime, they were unable to guess the weak associate. Thus, a simple strategy in which only one prediction of the target is made should produce no priming benefit on weak associate trials. However, a substantial effect was found.

More formally, if we assume that the generate subjects are relying on a simple predict-and-match strategy, we can estimate the predictability of weakly associated targets that is large enough to produce the magnitude of priming observed for these targets. We represent the probability of identification of the target under a simple predict-and-match strategy in terms of the probability of correctly generating the target, $\mathrm{P}(\mathrm{Gen})$, and the probability of identifying the target when it has or has not been correctly generated, $\mathrm{P}(\mathrm{Id} \mid \mathrm{Gen})$ or $\mathrm{P}(\mathrm{Id} \mid \sim$ Gen $)$, respectively:

$\mathrm{P}(\mathrm{Id})=\mathrm{P}($ Gen $) \cdot \mathrm{P}(\mathrm{Id} \mid$ Gen $)+\mathrm{P}(\sim \mathrm{Gen}) \cdot \mathrm{P}(\mathrm{Id} \mid \sim$ Gen $)$.

$\mathbf{P}(\mathrm{Id})$ is estimated from the identification probability for related targets with weak associates (.69); $\mathrm{P}(\mathrm{Id} \mid \mathrm{Gen})$ is set at its maximum of $1 ; \mathrm{P}(\sim \mathrm{Gen})=1-\mathrm{P}(\mathrm{Gen})$; and $\mathrm{P}(\mathrm{Id} \mid \sim$ Gen) is estimated from accuracy on new-target trials (.36).

We substituted the above values into the equation to solve for the predictability, $\mathrm{P}(\mathrm{Gen})$, of weakly associated targets, and obtained a value of .52 . This result is more than 17 times larger than the prediction accuracy achieved by the guess group for weakly associated targets (.03).

A second source of evidence, suggesting that the subjects generating an associate of the prime were not employing a simple predict-and-match strategy, comes from a comparison of intrusion errors for the two prime-task groups. The groups had similar means for the number of words erroneously given as the target during priming (4.9 and 5.7 for generate and read, respectively). All intrusions for the two groups were classified as associatively or semantically related to the prime, or unrelated, by the first author. Intrusions were judged to be related if they were common associates of the prime, or obviously shared a synonymy, category, or other semantic relationship with the prime (e.g., BREAD-LOAF, SUNBURN-RED, HEAVENGOD, SCRATCH-BACK, STEAM-KETTLE, BLOOM-BUD). The proportion of related intrusions was calculated for each subject. The important result of this analysis was that there was no difference between the groups in the mean proportion of intrusions that were semantically related to the prime (generate: $48 \%$, read: $53 \%$ ). Because only a very small proportion of related intrusions resembled the target visually or phonologically, it appears that many of the related intrusions were guesses made when the subjects had little perceptual information about the target.

\section{EXPERIMENT 3}

Experiment 2 failed to clarify the uncertainty arising in the first study about the presence of inhibitory effects in priming, and the relative size of such effects in the generate and read conditions. Experiment 3, involving only the read and generate groups, used two tests to address the issue of inhibition and the more general issue of strategies in using prime information. The first test was a set of "baseline" trials in which the targets, matched on frequency and length distribution to the targets used for priming, were preceded by a " + " warning signal instead of a prime. This method of presentation follows that of the duration determination phase, which immediately preceded these baseline trials.

The second test provided priming trials that were designed to be sensitive to the differential use of prediction or associative processing in the two groups. The targets on these test trials were replaced by visually similar foil words, in order to assess the extent to which the subjects would misidentify a foil (e.g., LATTICE) as a target (LETTUCE) after a related prime (GREEN) (cf. Norris, 1984; O'Connor \& Forster, 1981). In the conditions of stimulus uncertainty prevailing in the perceptual identification task, such a mispriming effect is likely to be large.

After the priming phase, the two groups had a repetition test on the primes, as in Experiment 1. The cuedrecall test, which consistently showed better memory for primes in the generate group in the two earlier studies, was dropped. This omission was necessary, because the inclusion of the priming trials necessary for the cued-recall test would approximately double the number of priming trials that were used in Experiments 1 and 2. Under these circumstances, interference from weak associations between cues with other items in the priming set is likely, and the cued-recall test was expected to elicit levels of recall too low to allow detection of a difference between the groups. The vulnerability of cued recall to the presence of weak cue-target associations has been demonstrated by Humphreys and Galbraith (1975).

To the extent that the generate task induces subjects to use information derived from the prime to identify the target, our intersection model predicts a greater inhibition on unrelated trials and a greater mispriming effect for the generate than the read group. The attentional account of Posner and Snyder (1975), and Becker's (1980) 
expectancy theory, allowing variation in the probability that subjects generate the expectancy set, would make the same prediction. A spread-of-activation account is broadly consistent with a mispriming effect, given that sometimes the node for the target would receive enough activation from the prime and the visually similar foil for it to be falsely recognized. However, it has no basis for predicting differential mispriming between the groups or any inhibitory effect.

\section{Method}

Subjects. Forty-eight introductory psychology students participated for course credit. They were randomly assigned to either the read or generate condition.

Materials and Design. The experiment began with the determination of stimulus duration in perceptual identification, as described previously, except that words were preceded by a neutral signal (" +"). A block of 24 trials at the selected duration was added to provide an assessment of baseline performance. The priming session followed. There were 32 related, 32 unrelated, 16 misprimed (prime-foil) trials, and 16 trials consisting of randomly re-paired primes and foils. The repetition test of primes was conducted last, in the same manner as the baseline trials.

Ninety-six paired associates were selected for the priming phase. Half of these pairs had been used in Experiment 2 and the remaining pairs were selected from word association norms (Palermo \& Jenkins, 1964; Postman \& Keppel, 1970; Thomson et al., 1976). The targets had a mean length of 4.8 letters (range 4-8) and a mean frequency of 131 (Kučera \& Francis, 1967). For the misprime trials, graphemically similar foils were devised by replacing or rearranging letters in each target. For $\mathbf{8 8}$ targets, one letter was replaced to produce a foil (e.g., HELL-HEAL, ROCKET-SOCKET), whereas for the remaining 8 targets, other changes were made (e.g., SILVERSLIVER, CHURCH-CHURNS). The length of the misprime foils was identical to that of the targets, but the foils were of lower frequency ( $M=39$, KuCera \& Francis).

Sixty words matched on mean length to the targets were used for duration determination. An additional 24 words, comparable in mean length and median frequency to the targets, were taken from the above association norms to serve as baseline items. For the repetition test, a further 48 words were selected from the association norms. These words were matched on mean and median frequency and mean length to the prime words and served as a pool from which distractors were taken. For each to-be-identified word, a pattern mask was prepared by randomly selecting eight uppercase consonants (excluding $\mathrm{Y}$ ), as described previously.

The items were counterbalanced over the four conditions for the priming phase. The 96 triples were divided into six subsets of 16 , matched approximately on mean target length. The subsets were rotated through the related target, unrelated target, misprimed (e.g., DREAM-STEEP), and unrelated foil (e.g., LAMP-STEEP) conditions. Unrelated target trials were randomly re-paired primes and targets, and unrelated foil trials were randomly re-paired primes and foils. The six subsets were combined into 6 different stimulus lists, each having four subsets prepared for the standard priming (two related, two unrelated) and two subsets prepared for mispriming (one misprimed, one unrelated). Over the 6 lists, each target and each foil occurred equally often in each of its two prime conditions. No target or foil was repeated in a list, and each target or its corresponding foil (but not both) appeared in each list. Two random sequences of pairs were prepared for each list, and 2 subjects from each group were run on each of the resulting 12 lists.

For the repetition test, half of the primes were chosen from each priming subset in each of the 12 priming lists to make up 12 sets of 48 primes corresponding to each of the 12 lists. The primes were selected such that each occurred six times in the 12 repetition lists, and representation of items from priming conditions was comparable to the priming phase. For each set, 24 new words were chosen from the pool of $\mathbf{4 8}$ distractor words such that each distractor occurred equally over the sets. The total list of 72 words was randomly sequenced.

Procedure. In the initial duration determination phase, the subjects saw 60 single words presented at a variable duration. On each trial, a warning signal (+) was presented in the center of the screen for $1 \mathrm{sec}$; after a 1-sec pause, the to-be-identified word was presented, followed immediately by a random-consonant mask for $100 \mathrm{msec}$. There was a pause of $4 \mathrm{sec}$ before the next trial. The stimuli were presented in blocks of 5 or 10 trials; typically two 5-trial blocks followed by five 10-trial blocks. The words were presented in the initial block at a duration of five frames (i.e., five 16.67-msec units, the frame length being determined by the refresh rate of the screen). The duration was decreased by one frame at the end of a block when accuracy was greater than or equal to $70 \%$, increased by one frame when accuracy was less than $30 \%$ for two consecutive blocks, and otherwise remained unchanged. Immediately after the 60 th trial, the 24 baseline trials were presented in two blocks of 12 trials at the stimulus duration determined for the subject.

The priming phase was conducted at the stimulus duration established during baseline. The procedure for priming was identical to the preliminary phase, except that the warning signal was replaced by the prime word on each trial. The subjects in the read group were instructed to read the prime word silently, whereas the subjects in the generate group were asked to silently generate an associate of the prime, as described previously. Ten practice trials with unrelated primes and targets were administered before the priming session. The final prime repetition phase was conducted in the same manner as the first phase, with primes and distractors presented one at a time after the warning signal.

The apparatus, presentation of stimuli, and instructions to the subjects were otherwise comparable to Experiments 1 and 2. The subjects were not informed about the misprime trials. Responses were scored by a computer keypress made by the experimenter, who also recorded on response sheets any incorrect identifications.

\section{Results}

The mean stimulus duration determined in the first phase was longer for the generate than the read group, but the difference was not significant [read $=46 \mathrm{msec}$, generate $=53 \mathrm{msec}, F(1,46)=3.7, p=.06$ ]. The baseline performance was significantly better for the generate group than for the read group in the items analysis $[F(1,23)=6.21, p<.05]$ but not the subjects analysis $[F(1,46)=1.71, p>.05]$. Mean accuracy on baseline trials and for the four conditions on priming trials is shown in Table 4. For standard priming, a group $\times$ prime type (related vs. unrelated) ANOVA revealed that there was a robust facilitation for related trials $[F(1,46)=218.15$ for subjects, and $F(1,95)=529.23$ for items, $p<.001]$. There was a marginally significant superiority for the generate group over the read group in the items analysis $[F(1,95)=3.90, p=.051]$ but not in the subjects analysis $(F<1)$. There was no group $\times$ prime interaction [ $F<1$ for subjects; $F(1,95)=1.95, p>.05$ for items]. A second analysis of priming compared the related prime with the baseline trials for the two groups. The results were similar, with prime type (related vs. neutral) having a substantial effect $[F(1,46)=37.46, p<.001$, for subjects; $F(1,118)=271.90, p<.001$, for items] and 
Table 4

Mean Proportions of Correct Identifications Over the Four Prime Types During Priming and for the Previously Presented Baseline Trials in Experiment 3

\begin{tabular}{lcccc} 
& \multicolumn{3}{c}{ Prime-Processing Group } \\
\cline { 2 - 3 } \cline { 5 - 5 } \multicolumn{1}{c}{ Condition } & $M$ & $S D$ & & \multicolumn{2}{c}{ Read } \\
\cline { 2 - 5 } \cline { 5 - 5 } Menerate & .10 & .15 & .12 \\
Misprimed Foil & .13 & .16 & .16 & .14 \\
Unrelated Foil & .16 & .16 & .13 & .15 \\
Related Target & .75 & .15 & .73 & .15 \\
Unrelated Target & .30 & .23 & .24 & .19 \\
Baseline & .61 & .15 & .55 & \\
\hline
\end{tabular}

no significant effect occurring for groups or the group $X$ prime type interaction.

An ANOVA comparing the two groups on the difference between baseline and unrelated prime trials assessed inhibitory effects of unrelated primes. In the subjects analysis, the trial type effect was reliable $[F(1,46)=100.04$, $p<.001]$, whereas the group and group $\times$ trial type effects were not. The trial type effect was confirmed in the items analysis $[F(1,118)=55.72, p<.001]$, and there was a group difference favoring the generate group $[F(1,118)=6.29, p<.05]$.

On misprime trials, the subjects performed poorly overall. A group $\times$ prime type (misprime vs. unrelated) ANOVA over subjects and items revealed no effect of group, prime, or their interaction. In order to assess the misleading effect of the primes, the error data were classified according to whether the foil was misidentified as its corresponding target. The proportions of these "false alarms" were .38 (generate) and .41 (read) on misprime trials, and .05 (generate) and .04 (read) on unrelated foil trials. An ANOVA confirmed that there was a significant mispriming effect in errors, with the subjects more likely to identify the foil as a target in the related than the unrelated condition [subjects, $F(1,46)=114.7$; items, $F(1,95)=161.36, p<.001]$. There was no significant group effect and importantly, no difference between the groups in the size of the mispriming effect $[F<$ and $F(1,95)=1.67, p>.05$ for the group $\times$ condition interaction for subjects and items, respectively].

In the prime repetition test, accuracy for primes was not significantly affected by priming phase condition; consequently, the data for these words were pooled over priming condition. A group $\times$ word type (old vs. new) ANOVA on accuracy confirmed a repetition benefit for old over new words [subjects: $F(1,46)=16.53, p<$ .001 ; items: $F(1,142)=5.89, p<.05]$. The important result, evident in Table 5, is that overall performance and the magnitude of the benefit were comparable for the two groups $(F \mathrm{~s}<1$ for the group main effect and the group $X$ word type interaction).

\section{Discussion}

The two groups performed similarly through the three phases of the experiment, except that the generate sub- jects tended to perform better overall in the baseline and priming phases. This effect was not reliable in any analysis by subjects and presumably reflects the fact that the generate subjects had (nonsignificantly) longer stimulus presentations than the read subjects.

The results replicate the priming effect and repetition benefit for the primes observed in the comparable groups in Experiments 1 and 2. As was the case in the first two experiments, the magnitude of these effects did not differ over the two prime-processing conditions.

Accuracy on misprime trials was nonsignificantly lower for related than unrelated targets. It was expected that performance would decline significantly on related trials if the subjects were being misled by the prime and identifying the foil as a target. The failure of this difference to reach significance may be a consequence of the very poor accuracy overall on misprime trials. This poor performance in turn is attributable to the lower frequency of the foils relative to targets. When error data were examined for false identifications of targets, a sizable and robust mispriming effect was observed. Approximately $40 \%$ of identification failures on related trials involved misidentification of the foil as its corresponding target. On the other hand, such misidentifications were rare on unrelated trials.

For the standard priming trials, there was evidence of an inhibitory effect on performance for unrelated targets. A comparison of unrelated targets with matched, previously presented, unprimed words revealed a significant accuracy decrement. The fact that the targets were different from the baseline items limits the interpretability of this effect; nevertheless, it is unlikely that item differences produced an effect of such magnitude (more than $40 \%$ ). In addition, accuracy on new words in the final repetition test of primes was considerably higher than on unrelated trials (means of $53 \%$ vs. $27 \%$, respectively, for the two groups combined). Consequently, the decrement is unlikely to be the result of fatigue or other changes occurring after the baseline phase of the experiment.

It is important that the above mispriming and inhibitory effects were comparable in magnitude for the two prime-processing groups. Consequently, Experiment 3 offers some clarification of the results of Experiments 1 and 2 , in which it was unclear whether the subjects generating an associate of the prime were showing greater inhibitory effects than the subjects reading the prime. The

Table 5

Mean Proportions of Correct Identifications in the Repetition Test for Old Primes (Pooled Over Related and Unrelated) and New Words in Experiment 3

\begin{tabular}{llllll}
\hline & \multicolumn{3}{c}{ Prime-Processing Group } \\
\cline { 2 - 3 } \cline { 5 - 6 } Word & \multicolumn{2}{c}{ Generate } & & \multicolumn{2}{c}{ Read } \\
\cline { 2 - 5 } Type & $M$ & $S D$ & & $M$ & $S D$ \\
\hline Old & .60 & .13 & & .60 & .14 \\
New & .52 & .14 & .54 & .19 \\
\hline
\end{tabular}


present results suggest that apparent differences between the two groups are not reliable. Rather, it appears that the subjects in the two conditions were using the prime to the same extent, to provide themselves with information about the identity of the target word.

\section{GENERAL DISCUSSION}

The results from Experiment 1 support Bentin and Katz's (1984) conjecture about reduced priming levels being caused by a lack of "whole word" or perceptual processing. Our experiment differed from Bentin and Katz's experiment in that we measured the extent to which primeprocessing tasks induced perceptual processing, instead of trying to infer it on intuitive grounds. We found that the asterisk-search subjects showed reduced priming that was accompanied by a reduced repetition effect for primes (our measure of perceptual processing) relative to the generate and read subjects. Nevertheless, the priming and repetition effects were significant for the asterisk-search subjects. Consequently, it is unclear whether there was reduced perceptual processing for all words or whether the subjects processed some primes fully and others inadequately. This question could be studied in a naming or lexical decision task by showing that there was a bimodal distribution of latencies in the related condition of the asterisk-search task. Clearly, our results suggest that the reduction in priming with superficial prime processing, as reported by Henik et al. (1983), Parkin (1979), and Smith et al. (1983), was due to inadequate perceptual processing. On the basis of the present results, we cannot determine whether the priming that was observed in the asterisk-search group was strategic or nonstrategic in nature.

The read and generate subjects in Experiment 1 showed comparable priming effects. In order to clarify the nature of priming in these groups, an additional, guessing group was employed in Experiment 2. Its purpose was to provide predictions of target identity over the unrelated, and weak and strong associate pairs used in priming. In the case of both weak and strong associates, the read and generate groups were comparable in the magnitude of priming they displayed. Furthermore, the probability of identification of targets with weak associates as primes was much higher than the probability with which these targets were produced by the guessing subjects. These results are inconsistent with a simple predict-and-match strategy in which a single prediction from the prime is matched with the target stimulus information. They also rule out a simple guessing account of priming in which subjects use the prime to generate a guess whenever the perceptual information available is inadequate for target identification.

In Experiment 3, we compared the read and generate groups on inhibition assessed against unprimed trials. In addition, reliance on the prime, which presumably is partly responsible for inhibitory effects, was assessed via the magnitude of mispriming. There was evidence of in- hibition and mispriming in both groups, and unequivocally, the groups were comparable on these measures, as well as on priming and on repetition benefits for the primes.

Although we can eliminate a simple guessing account of the priming data for the read and generate groups, it is also necessary to consider a more sophisticated guessing model in which the subject uses the prime to continuously generate words until one matches the partial perceptual information extracted from the target, or uses partial perceptual information about the target to continuously generate words until one is related to the prime. Both of these alternatives seem unlikely, particularly for the weakly related targets in Experiment 2 that were correctly predicted on the first guess only $3 \%$ of the time. In addition, although a substantial proportion of intrusion errors in the read and generate groups over the three experiments were semantically related to the prime (average $43 \%$ ), or visually similar to the target (average $28 \%$ ), the overall number of intrusions was quite low $(18 \%$ of errors). The low intrusion rate is compatible with a process that combines information from the prime and target prior to retrieval, instead of trying to use available retrieval information to make a "best" guess.

The adequacy of sophisticated guessing accounts can also be questioned on the basis of a memory research finding discussed by Humphreys et al. (1989). The result was reported in an investigation by Graf and Schacter (1985), who had amnesics and other subjects study pairs of unrelated words. These subjects were then given a cue word and the first three letters of the target word and asked to produce the first word that came to mind that completed the stem. The presence of the study cue at the time of test enhanced performance relative to the presence of another unrelated word. In this task, a strategy of generating to the cue term would be ineffective because the probability of generating the target term was essentially zero, and a strategy of generating to the stem would produce words that were unrelated to the cue. Furthermore, amnesic subjects would not have been able to use the more beneficial strategy of checking the episodic appropriateness of the words that they generated by trying to recognize whether they had been paired with the cue word during study. Humphreys et al. (1989) concluded that the cue and the stem were not operating independently to affect retrieval. Instead, they were combining to increase the probability that the target word was the first word produced.

Returning to the discussion of the basis of priming effects in the read and generate groups, we find that the three experiments together provide considerable information on this issue. First, there was a difference in cued recall (generate superior to read: Experiments 1 and 2), indicating that we had been successful in producing differences in the amount of semantic or elaborative processing of the primes. However, there was no difference in priming (Experiments 1,2, and 3) and no difference in the repetition effect (Experiments 1 and 3 ) between the 
generate and read groups. Furthermore, as noted above, the comparable performance of the read and generate subjects also held for priming with strong and weak associates (Experiment 2) and for inhibition and mispriming (Experiment 3).

Contrary to the results of Parkin $(1979,1984)$ and Smith et al. (1983), variations in depth-of-prime processing were detected in the conceptually driven cued-recall test, but not in priming in perceptual identification. Despite the importance of the associative relation between prime and target, semantic priming in perceptual identification resembles the repetition effect in its sensitivity to variations in perceptual, rather than conceptual, processing. A recent report of item relatedness effects by Besson, Fischler, Boaz, and Raney (1992) shows a similar dissociation between levels of processing and associative effects. Benefits in cued recall and fragment completion for previously presented associative pairs were attributed to associative activation occurring during a study task involving graphemic judgments about pair members. These benefits were independent of a levels-of-processing benefit observed for the graphemic versus a semantic study task in the cued-recall test and an episodic recognition test. Thus, semantic priming in perceptual identification and perhaps other word recognition tasks appears to reflect the operation of associative processing that is readily elicited and is less effortful than the processing required for the present generate task and other semantic tasks. A related distinction between automatic, nonanalytic, and intentional analytic memory processes has been made by Jacoby (1991).

The present results have an obvious implication for depth-of-prime-processing investigations in lexical decision, naming, and color-naming tasks. Because we found better episodic memory for primes in the generate than the read group, but no difference between the groups in the magnitude of priming, mispriming, and inhibition, we can reject the tentative generalization made from previous studies (Parkin, 1979, 1984; Smith et al., 1983) that primeprocessing manipulations have common effects on episodic and semantic memory tasks. It appears that although deeper or more elaborative prime processing enhances episodic memory, elaborative processing per se does not affect priming. This claim is consistent with a recent study we conducted on the pronunciation task (Burt \& Mardle, 1990), in which deeper prime processing produced better episodic memory for primes, but produced weaker priming than did reading the primes.

The priming and memory results for the read and generate conditions are in conflict with the results obtained in lexical decision by Smith et al. (1983) for read versus semantic prime-processing tasks. Those authors reported that the semantic task produced better episodic memory for primes and stronger priming than the read task. It is not difficult to find explanations for the difference between the Smith et al. and the present study in the priming for the read condition. First, because Smith et al. did not assess repetition effects for primes, it is possible that the subjects in the read condition were not reading primes consistently. However, the relatively high episodic recognition performance on primes for the subjects in this condition casts doubt on this possibility. It is more likely that, as suggested in the introduction, subjects show greater priming effects when the characteristics of the task encourage reliance on the prime. In the perceptual identification task, there is more uncertainty about the identity of the target and it is not surprising that subjects show strong priming effects when required only to read the prime silently.

There is little support in the results of the read and generate groups for a spread-of-activation or other "automatic" priming process, because reading of the prime produced substantial priming in the present studies but not in the lexical decision experiments of Smith et al. (1983). In addition, the substantial inhibitory effect on unrelated trials observed in Experiment 3 is inconsistent with the spread-of-activation theory, which allows for preactivation of nodes of words related to the prime but no effect on nodes for unrelated words.

A more successful account of the priming effect for read and generate groups is a prospective, expectancy model of the kind proposed by Posner and Snyder (1975), Becker (1980), or Humphreys et al. (1992). That is, subjects generate some expectancy about the target at the time of prime processing, with a consequent benefit in recognition of related targets and a cost in the recognition of unrelated targets. In order to explain the magnitude of the priming effects with weak associates in Experiment 2, the expectancy must be construed as a set of semantic attributes or words related to the prime, rather than a single prediction. The present results complement recent evidence for expectancy-based priming in the pronunciation task (Burt, Humphreys, \& Mardle, 1990; Keefe \& Neely, 1990). Because naming and perceptual identification are not susceptible to response bias effects, for example, arising from the operation of a hypothesized "meaning integration" or other strategy in lexical decision, these results are readily interpreted in terms of prospective rather than retrospective mechanisms.

The sequential intersection model of priming (Humphreys et al., 1992) explains the priming data in terms of associative processing of the prime by the read and generate subjects. The present results do not distinguish the intersection model from Becker's (1980) theory, although the intersection model makes different proposals about the way in which prime-generated expectancies are combined with perceptual information about the target. In Becker's model, the members of the expectancy set generated from the prime are matched with the target input in a terminating, serial search; whereas the intersection process, operating within a connectionist framework, finds in a single step the item(s) common to the word sets activated by the prime and the target. Humphreys et al. (1992) have shown that the Ratcliff and McKoon (1988) compound cue model is structurally similar to the sequential intersection model, although the conceptual frame- 
work differs in the two models. The present framework allows the compound cue model to be applied to naming and perceptual identification, instead of being restricted to decision tasks (cf. Neely, 1991). Also, in contrast to the compound cue model, the present model conceives of priming as prospective, and interprets inhibitory effects on unrelated trials by supposing that subjects do not use the intersection as a basis for target identification when the prime is a neutral, nonword stimulus.

The sequential intersection model provides a useful account of depth-of-prime-processing effects because it deals in a principled way with task differences in priming and prime processing. The intersection process in the sequential intersection model is conceived as a process commonly relied upon in memory retrieval when contextual information is available to enable more focused and less noisy memory access. The extent to which subjects employ associative prime processing, and the weight given to information from the prime in the intersection process, vary with task demands. Thus, in the pronunciation task, in which the target is presented in the clear and the informational value of the prime is relatively low, priming (and inhibitory) effects are small. In the lexical decision task, and to a greater extent in perceptual identification, the information provided by the prime is more valuable and priming effects are larger. Within tasks, manipulations that increase the predictive validity of the prime should increase associative processing of the prime, with a consequent benefit in conceptual memory tests of the prime without any change in perceptual effects, such as repetition of the prime in perceptual identification. Precisely this result was observed by Burt et al. (1990) in an experiment demonstrating an increase in priming in pronunciation with an increase in the proportion of related trials.

In conclusion, we suggest that in perceptual identification, subjects respond to target ambiguity and the presence of a substantial number of strongly related primetarget pairs by attempting to produce a word that fits the perceptual input and is related to the prime. The sequential intersection model describes a direct access procedure for accomplishing this task. This procedure is considered (Humphreys et al., 1992) to be a general mechanism for identifying a word against a noisy background and, as such, has application to priming and other memory access effects within the context of reading and memory outside the laboratory.

\section{REFERENCES}

BADDELEY, A. (1990). Human memory: Theory and practice. Hillsdale, NJ: Erlbaum.

BECKER, C. A. (1979). Semantic contexts and word frequency effects in word recognition. Journal of Experimental Psychology: Human Perception \& Performance, 5, 252-259.

BeCKer, C. A. (1980). Semantic context effects in visual word recognition: An analysis of semantic strategies. Memory \& Cognition, 8 , 493-512.

BECKER, C. A. (1985). What do we really know about semantic context effects in reading? In D. Besner, T. G. Waller, \& E. M. Mac-
Kinnon (Eds.), Reading research: Advances in theory and practice (Vol. 5, pp. 125-166). Toronto: Academic Press

Bentin, S., \& Katz, L. (1984). Semantic awareness in a nonlexical task. Bulletin of the Psychonomic Society, 22, 381-384.

Besner, D. Smith, M. C., \& MacLeod, C. M. (1990). Visual word recognition: A dissociation of lexical and semantic processing. Journal of Experimental Psychology: Learning, Memory, \& Cognition, 16, 862-869.

Besson, M., Fischler, I., Boaz, T., \& Raney, G. (1992). Effects of automatic associative activation on explicit and implicit memory tests. Journal of Experimental Psychology: Learning, Memory, \& Cognition, 18, 89-105.

Burke, D. M., White, H., \& Diaz, D. L. (1987). Semantic priming in young and older adults: Evidence for age constancy in automatic and attentional processes. Journal of Experimental Psychology: $\mathrm{Hu}$ man Perception \& Performance, 13, 79-88.

Burt, J. S., Humphreys, M. S., \& Mardle, L. (1990). Associative prime processing in the pronunciation task. Unpublished manuscript, University of Queensland.

BuRT, J. S., \& MARDLE, L. (1990). Depth of exprime-processing effects in pronunciation. Unpublished manuscript, University of Queensland.

Collins, A. M., \& Lorus, E. F. (1975). A spreading activation theory of semantic processing. Psychological Review, 82, 407-428.

Craik, F. I. M., \& LockHarT, R. S. (1972). Levels of processing: A framework for memory research. Journal of Verbal Learning \& Verbal Behavior, 11, 671-684.

Craik, F. I. M., \& Tulving, E. (1975). Depth of processing and retention of words in episodic memory. Journal of Experimental Psychology: General, 104, 268-294.

DE Groot, A. M. B. (1984). Primed lexical decision: Combined effects of the proportion of related prime-target pairs and the stimulusonset asynchrony of prime and target. Quarterly Journal of Experimental Psychology, 36A, 253-280.

DE Groot, A. M. B. (1985). Word-context effects in word naming and lexical decision. Quarterly Journal of Experimental Psychology, 37A, 281-297.

de Groot, A. M. B., Thomassen, A. J. W. M., \& Hudson, P. T. W. (1982). Associative facilitation of word recognition as measured from a neutral prime. Memory \& Cognition, 10, 358-370.

DEN HEYER, K. (1985). On the nature of the proportion effect in se mantic priming. Acta Psychologica, 60, 25-38.

den Heyer, K., Briand, K., \& Sith, L. (1985). Automatic and strategic effects in semantic priming: An examination of Becker's verification model. Memory \& Cognition, 13, 228-232.

EvetT, L. J., \& HUMPhreYs, G. W. (1981). The use of abstract graphemic information in lexical access. Quarterly Joumal of Experimental Psychology, 33, 325-350.

Fischler, I., \& Goodman, G. O. (1978). Latency of associative activation in memory. Joumal of Experimental Psychology: Human Perception \& Performance, 4, 455-470.

FORSTER, K. I., \& DAVIS, C. (1984). Repetition priming and frequency attenuation in lexical access. Journal of Experimental Psychology: Learning, Memory, \& Cognition, 10, 680-698.

Graf, P., \& Schacter, D. L. (1985). Implicit and explicit memory for new associations in normal and amnesic subjects. Journal of Experimental Psychology: Leaming, Memory, \& Cognition, 11, 501-518.

HAYMAN, C. A., \& JACOBY, L. L. (1989). Specific word transfer as a measure of processing in the word-superiority paradigm. Memory \& Cognition, 17, 125-133.

Henik, A., Friedrich, F. J., \& Kelloga, W. A. (1983). The dependence of semantic relatedness effects upon prime processing. Memory \& Cognition, 11, 366-373.

Humphreys, M. S., Bain, J. D., \& Pike, A. R. (1989). Different ways to cue a coherent memory system: A theory for episodic, semantic and procedural tasks. Psychological Review, 96, 208-231.

Humphreys, M. S., Bain, J. D., Sweeton, J. J., \& Pacey, P. M. (1991). Encoding specificity II: Is transfer appropriate processing sufficient? Unpublished manuscript, University of Queensland.

Humphreys, M. S., \& Galbraith, R. C. (1975). Forward and backward associations in cued recall: Predictions from the encoding speci- 
ficity principle. Journal of Experimental Psychology: Human Learning \& Memory, 1, 702-710.

Humphreys, M. S., Wiles, J., \& Bain, J. D. (1992). Memory retrieval with two cues: Think of intersecting sets. In D. E. Meyer \& S. Kornblum (Eds.), Attention and Performance XIV: A silver jubilee. Cambridge, MA: MIT Press.

JACOBY, L. L. (1983). Remembering the data: Analyzing interactive processes in reading. Journal of Verbal Leaming \& Verbal Behavior. $22,485-508$.

JACOBY, L. L. (1991). A process dissociation framework: Separating automatic from intentional uses of memory. Journal of Memory \& Language, 30, 513-541.

JACOBY, L. L., \& DALLAS, M. (1981). On the relationship between autobiographical memory and perceptual learning. Journal of Experimental Psychology: General, 110, 306-340.

JACOBY, L. L., \& WITHERSPOON, D. (1982). Remembering without awareness. Canadian Journal of Psychology, 36, 300-324.

KAYE, D. B., \& BRoWN, S. W. (1985). Levels and speed of processing effects on word analysis. Memory \& Cognition, 13, 425-434.

Keefe, D. E., NeELY, J. H. (1990). Semantic priming in the pronunciation task: The role of prospective prime-generated expectancies. Memory \& Cognition, 18, 289-298.

Kirsner, K., Milech, D., \& Standen, P. (1983). Common and modality-specific processes in the mental lexicon. Memory \& Cognition, 11, 62!-630.

KORIAT, A. (1981). Semantic facilitation in lexical decision as a function of prime-target association. Memory \& Cognition, 9, 587-598.

KuČera, H., \& Francis, W. N. (1967). Computational analysis of present-day American English. Providence, RI: Brown University Press.

Lorch, R. F., Balota, D. A., \& Stamm, E. G. (1986). Locus of inhibition effects in lexical decisions: Pre- or postlexical access? Memory \& Cognition, 14, 95-103.

Meyer, D. E., \& Schvaneveldt, R. W. (1971). Facilitation in recognising pairs of words: Evidence of a dependence between retrieval operations. Journal of Experimental Psychology, 90, 227-234.

Miller, K. M. (1970). Free-association responses of English and Australian students to 100 words from the Kent-Rosanoff word association test. In L. Postman \& G. Keppel (Eds.), Norms of word association. New York: Academic Press.

Morris, C. D., Bransford. J. D., \& Franks, J. J. (1977). Levels of processing versus transfer appropriate processing. Journal of Verbal Learning \& Verbal Behavior, 16, 519-533.

NeELY, J. H. (1977). Semantic priming and retrieval from lexical memory: Roles of inhibitionless spreading activation and limited capacity attention. Journal of Experimental Psychology: General, 106, 226254.

NeELY, J. H. (1991). Semantic priming effects in visual word recognition: A selective review of current findings and theories. In D. Besner \& G. Humphreys (Eds.), Basic processes in reading: Visual word recognition. Hillsdale, NJ: Erlbaum.

Nelson, D. L., \& MCEvoy, C. L. (1979). Encoding context and set size. Joumal of Experimental Psychology: Human Leaming \& Memory, 5, 292-314.

NoRRIS, D. (1984). The mispriming effect: Evidence of an orthographic check in the lexical decision task. Memory \& Cognition, 12, 470-476.

O'CONNOR, R. E., \& FORSTER, K. I. (1981). Criterion bias and search sequence bias in word recognition. Memory \& Cognition, 9, 78-92.
Palermo, D. S., \& Jenkins, J. J. (1964). Word association norms: Grade school through college. Minneapolis: University of Minnesota Press.

Parkin, A. J. (1979). Specifying levels of processing. Quarterly Joumal of Experimental Psychology, 31, 175-195.

PARKIN, A. J. (1984). Levels of processing, context, and the facilitation of pronunciation. Acta Psychologica, 55, 19-29.

Posner, M. 1., \& S Nyder, C. R. R. (1975). Attention and cognitive control. In R. L. Solso (Ed.), Information processing and cognition: The Loyola symposium. Hillsdale, NJ: Erlbaum.

Postman, L., \& Keppel, G. (EDs.). (1970). Norms of word association. New York: Academic Press.

RatClifF, R., McKoon, G. (1988). A retrieval theory of priming in memory. Psychological Review, 95, 385-408.

Roediger, H. L., III, Weldon, M. S., Chaldis, B. H. (1989). Explaining dissociations between implicit and explicit measures of retention: A processing account. In H. L. Roediger, Ill \& F. I. M. Craik (Eds.), Varieties of memory and consciousness: Essays in Honour of Endel Tulving. Hillsdale, NJ: Erlbaum.

Scarborough, D. L., Cortese, C., \& Scarborough, H. S. (1977). Frequency and repetition effects in lexical memory. Jourmal of Experimental Psychology: Human Perception \& Performance, 3, 1-17.

Seidenberg, M. S., Waters, G. S., Sanders, M., Langer, P. (1984). Pre- and postlexical loci of contextual effects on word recognition. Memory \& Cognition, 12, 315-328.

Smiтh, M. C. (1979). Contextual facilitation in a letter search task depends on how the prime is processed. Journal of Experimental Psychology: Human Perception \& Performance, 5, 239-251.

Smith, M. C., Theodor, L., \& Franki.j, P. E. (1983). The relationship between contextual facilitation and depth of processing. Journal of Experimental Psychology: Learning, Memory \& Cognition, 9, 697-712.

Stanovich, K. E., \& West, R. F. (1983). On priming by sentence context. Journal of Experimental Psychology: General, 112, 1-36.

Thomson, D. M., Meredith, S. G., \& Browning, C. (1976). Monash free association norms. Monash University.

TWEEDY, J. R., \& LAPINSKI, R. H. (1981). Facilitating word recognition: Evidence for strategic and automatic factors. Quarterly Journal of Experimental Psychology, 33A, 51-59

TWEeDY, J. R., LAPINSKI, R. H., \& SCHVANEVELDT, R. W. (1977). Semantic-context effects on word recognition: Influence of varying the proportion of items presented in an appropriate context. Memory \& Cognition, $5,84-89$.

WARREN, R. E. (1974). Association, directionality, and stimulus encoding. Journal of Experimental Psychology, 94, 90-100.

\section{NOTE}

1. It was discovered after completion of the first two experiments that the monitor used for stimulus presentation had a scan rate that precluded adjustments of less than $16.7 \mathrm{msec}$ in presentation duration. Consequently, there are unknown variations from the specified duration set for each subject. This limitation was taken into account in Experiment 3.

(Manuscript received August 8, 1989; revision accepted for publication May 28, 1992.) 